\title{
Population Data on Finland 1900-1979
}

\section{Resident population on 31 December}

\begin{tabular}{|c|c|c|c|c|c|}
\hline & Thole country & \multicolumn{2}{|c|}{$\begin{array}{l}\text { Urban municipalities } \\
\text { number }\end{array}$} & \multicolumn{2}{|c|}{$\begin{array}{c}\text { Rural municipalities } \\
\text { number }\end{array}$} \\
\hline 1900 & 2655900 & 333300 & 12.5 & 2322600 & 87.5 \\
\hline 1910 & 2943400 & 432200 & 14.7 & 2511200 & 85.3 \\
\hline 1920 & 3147600 & 507400 & 16.1 & 2640200 & 83.9 \\
\hline 1930 & 3462700 & 715000 & 20.6 & 2747700 & 79.4 \\
\hline 1940 & 3695600 & 991700 & 26.8 & 2703900 & 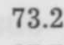 \\
\hline 1950 & 4029800 & 1302400 & 32.3 & 2727400 & 7 \\
\hline 1960 & 4446200 & 1707000 & 38.4 & 2739200 & t \\
\hline 1965 & 4569900 & 2016000 & 44.1 & 2553900 & 55. \\
\hline 1970 & 4598300 & 2340300 & 50.9 & 2258000 & 49. \\
\hline 1975 & 4715804 & 2776047 & 58.9 & 1939996 & 41. \\
\hline 1976 & 4732973 & 2793004 & 59.0 & 1949868 & 41. \\
\hline 1977 & 4746967 & 2834162 & 59.7 & 1912805 & 40. \\
\hline 1978 & 4758088 & 2840574 & 59.7 & 1917514 & 40. \\
\hline 1979 & 4771098 & 2851582 & 59.8 & 1919516 & 40. \\
\hline
\end{tabular}

\section{Age Structure}

\begin{tabular}{|c|c|c|c|c|c|c|c|c|c|}
\hline & Who & e popul & tion & & Men & & & Women & \\
\hline & $\frac{0-14}{\%}$ & $\begin{array}{c}15-64 \\
\%\end{array}$ & $\frac{65-}{\%}$ & $\frac{0-14}{\%}$ & $\begin{array}{c}15-64 \\
\%\end{array}$ & $\frac{65-}{\%}$ & $\frac{0-14}{\%}$ & $\begin{array}{c}15-64 \\
\%\end{array}$ & $\frac{65-}{0 \%}$ \\
\hline 1900 & 35.0 & 59.6 & 5.4 & 35.9 & 59.4 & 4.7 & 34.3 & 59.7 & 6.0 \\
\hline 1910 & 35.6 & 58.6 & 5.8 & 36.7 & 58.3 & 5.0 & 34.6 & 59.0 & 6.4 \\
\hline 1920 & 33.4 & 60.7 & 5.9 & 34.8 & 60.0 & 5.2 & 32.0 & 61.3 & 6.7 \\
\hline 1930 & 29.4 & 64.3 & 6.3 & 30.6 & 64.1 & 5.3 & 28.3 & 64.5 & 7.2 \\
\hline 1940 & 26.9 & 66.7 & 6.4 & 28.2 & 66.5 & 5.3 & 25.7 & 66.9 & 7.4 \\
\hline 1950 & 30.0 & 63.3 & 6.7 & 32.0 & 62.8 & 5.2 & 28.1 & 63.9 & 8.0 \\
\hline 1960 & 30.1 & 62.4 & 7.4 & 31.9 & 62.3 & 5.7 & 28.5 & 62.5 & 8.9 \\
\hline 1965 & 26.7 & 65.3 & 8.0 & 28.2 & 65.6 & 6.2 & 25.4 & 64.9 & 9.7 \\
\hline 1970 & 24.4 & 66.3 & 9.3 & 25.7 & 67.1 & 7.2 & 23.0 & 65.7 & 11.3 \\
\hline 1975 & 21.9 & 67.6 & 10.5 & 23.1 & 68.7 & 8.2 & 20.7 & 66.2 & 13.1 \\
\hline 1976 & 21.5 & 67.4 & 11.1 & 22.7 & 68.8 & 8.4 & 20.3 & 66.1 & 13.6 \\
\hline 1977 & 21.2 & 67.4 & 11.4 & 22.4 & 69.0 & 8.6 & 20.1 & 65.9 & 14.0 \\
\hline 1978 & 20.8 & 67.5 & 11.7 & 22.0 & 69.2 & 8.8 & 19.7 & 65.9 & 14.4 \\
\hline
\end{tabular}




\section{Live births}

$\begin{array}{lcc} & \text { Number } & \begin{array}{c}\text { Per } 1000 \\ \text { of mean } \\ \text { population }\end{array} \\ 1900-10 & 90293 & 32.4 \\ 1911-20 & 83045 & 27.0 \\ 1921-30 & 78184 & 23.6 \\ 1931-40 & 70584 & 19.7 \\ 1941-50 & 92613 & 24.3 \\ 1951-60 & 88025 & 20.7 \\ 1961-70 & 76466 & 16.8 \\ 1971-75 & 60982 & 13.1 \\ 1975 & 65719 & 13.9 \\ 1976 & 66846 & 14.0 \\ 1977 & 65659 & 13.9 \\ 1978 & 63983 & 13.4 \\ 1979 & 63475 * & 13.3\end{array}$

\section{Age specific fertility rate}

Per 1000 women

Total

fertility

15-19 20-24 25-29 $30-34 \quad 35-39 \quad 40-44 \quad 45-49$ per woman

$\begin{array}{lrrrrrrrr}1901-10 & 17.6 & 157.8 & 213.0 & 236.9 & 191.0 & 114.4 & 18.2 & 4.68 \\ 1911-20 & 14.9 & 122.8 & 181.4 & 181.9 & 155.2 & 92.9 & 14.0 & 3.81 \\ 1921-30 & 14.2 & 110.0 & 156.9 & 143.8 & 116.8 & 67.4 & 10.5 & 3.10 \\ 1931-40 & 14.4 & 96.5 & 126.2 & 108.9 & 82.8 & 43.5 & 5.9 & 2.39 \\ 1941-45 & 11.3 & 105.2 & 144.9 & 124.3 & 88.4 & 41.8 & 5.4 & 2.60 \\ 1946-50 & 25.7 & 161.9 & 189.4 & 147.5 & 100.5 & 43.3 & 5.0 & 3.37 \\ 1951-55 & 27.2 & 157.6 & 165.8 & 125.0 & 81.0 & 35.1 & 3.9 & 2.98 \\ 1956-60 & 29.3 & 161.4 & 159.6 & 108.1 & 67.5 & 27.5 & 2.8 & 2.78 \\ 1961-65 & 30.7 & 156.7 & 156.0 & 98.8 & 55.7 & 22.5 & 2.2 & 2.61 \\ 1966-70 & 34.7 & 131.9 & 125.9 & 76.5 & 39.7 & 13.7 & 1.4 & 2.12 \\ 1971-75 & 27.9 & 104.3 & 106.6 & 55.8 & 23.3 & 6.1 & 0.5 & 1.62 \\ 1975 & 27.5 & 105.5 & 113.9 & 59.6 & 24.7 & 5.9 & 0.4 & 1.69 \\ 1976 & 26.1 & 103.8 & 116.3 & 65.6 & 25.2 & 5.7 & 0.5 & 1.72 \\ 1977 & 24.2 & 99.2 & 115.2 & 67.8 & 25.6 & 5.9 & 0.4 & 1.69 \\ 1978 & 21.3 & 95.5 & 113.7 & 67.4 & 26.5 & 5.7 & 0.3 & 1.65\end{array}$

Illigitimate live births

$1901-10$
$1911-20$
$1921-30$
$1931-40$
$1941-50$
$1951-60$
$1961-70$
$1971-75$

6120

6573

6606

5346

5629

3819

3621

4824

Per cent of all live births
6.8
6.8
8.4
7.6
6.1
4.3
4.7
7.9
(cont.) 
(cont.)

1975

$\begin{array}{ll}6670 & 10.1 \\ 7273 & 10.9 \\ 7317 & 11.1 \\ 7263 & 11.4\end{array}$

\section{Abortions}

$\begin{array}{lcc} & \text { Abortions } & \begin{array}{c}\text { Abortions per } 100 \\ \text { live births }\end{array} \\ 1971 & 20622 & 33.8 \\ 1972 & 22146 & 37.5 \\ 1973 & 23217 & 40.9 \\ 1974 & 22846 & 36.5 \\ 1975 & 21547 & 32.8 \\ 1976 & 19818 & 29.6 \\ 1977 & 17772 & 27.1 \\ 1978 & 16928 & 26.4\end{array}$

Children per 1000 women living in 1970 and born in 1906-36, by socio-economic status

\section{Children/women}

1. All women

2.52

2. Employers

2.1. Farmer employers

3.09

2.2. Other employers

2.53

3.30

3.46

3.1. Farmers on own-account

4. Managers and senior administrative or clerical employees

4.1. Government administrators and executive officials

4.2. Commercial managers

4.3 Other senior administrative or clerical employees

5. Junior administrative or clerical employees

6. Skilled or specialized workers

6.1. Farm and forestry workers

7. Labourers

8. Students

9. Others 
Gross and Net Reproduction Rate

$\begin{array}{lcc} & \begin{array}{c}\text { Gross Reproduction } \\ \text { Rate }\end{array} & \begin{array}{c}\text { Net Reproduction } \\ \text { Rate }\end{array} \\ 1901-10 & 2.278 & \ldots \\ 1911-20 & 1.849 & \ldots \\ 1921-30 & 1.504 & \ldots \\ 1931-35 & 1.167 & 0.958 \\ 1936-40 & 1.162 & 0.962 \\ 1941-45 & 1.262 & 1.048 \\ 1946-50 & 1.637 & 1.469 \\ 1951-55 & 1.452 & 1.373 \\ 1956-60 & 1.357 & 1.301 \\ 1961-65 & 1.276 & 1.236 \\ 1966-70 & 1.035 & 1.009 \\ 1971-75 & 0.790 & 0.770 \\ 1975 & 0.819 & 0.798 \\ 1976 & 0.835 & 0.814 \\ 1977 & 0.825 & 0.804 \\ 1978 & 0.804 & 0.787\end{array}$

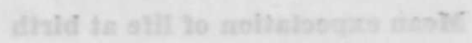

\section{Marriages}

$\begin{array}{lcc} & \text { Number } & \begin{array}{c}\text { per } 1000 \text { of mean } \\ \text { population }\end{array} \\ 1901-10 & 18947 & 6.8 \\ 1911-20 & 18922 & 6.2 \\ 1921-30 & 23725 & 7.2 \\ 1931-40 & 28490 & 7.9 \\ 1941-50 & 37367 & 9.5 \\ 1951-60 & 32141 & 7.6 \\ 1961-70 & 37398 & 8.2 \\ 1971-75 & 34871 & 7.5 \\ 1975 & 31547 & 6.7 \\ 1976 & 32004 & 6.8 \\ 1977 & 30966 & 6.5 \\ 1978 & 29760 & 6.2 \\ 1979 & 29324 * & 6.1\end{array}$


Deaths

$\begin{array}{lccc}\text { Number } & \begin{array}{c}\text { per } 1000 \\ \text { of mean } \\ \text { population }\end{array} & \begin{array}{c}\text { Infant mortality } \\ \text { per } 1000 \text { live } \\ \text { births }\end{array} \\ 1901-10 & 52099 & 18.7 & 123.8 \\ 1911-20 & 58284 & 18.9 & 111.8 \\ 1921-30 & 49572 & 14.9 & 91.9 \\ 1931-40 & 50229 & 14.0 & 72.0 \\ 1941-50 & 51837 & 13.6 & 56.0 \\ 1951-60 & 39380 & 9.3 & 28.6 \\ 1961-70 & 43493 & 9.5 & 16.7 \\ 1971-75 & 44351 & 9.5 & 11.3 \\ 1975 & 43828 & 9.3 & 10.0 \\ 1976 & 44786 & 9.5 & 9.9 \\ 1977 & 44605 & 9.3 & 9.1 \\ 1978 & 43692 & 9.1 & 7.6 \\ 1979 & 43737 * & 9.1 & \ldots\end{array}$

Mean expectation of life at birth

$\begin{array}{lcc} & \text { Men } & \text { Women } \\ 1911-200^{1} & 43.4 & 49.1 \\ 1921-30 & 50.7 & 55.1 \\ 1931-40^{2} & 54.5 & 59.6 \\ 1941-45^{2} & 54.6 & 61.1 \\ 1946-50 & 58.6 & 65.8 \\ 1951-55 & 63.4 & 69.8 \\ 1956-60 & 64.9 & 71.6 \\ 1961-65 & 65.4 & 72.6 \\ 1966-70 & 65.9 & 73.6 \\ 1971 & 65.9 & 74.2 \\ 1972 & 66.6 & 74.9 \\ 1973 & 66.9 & 75.5 \\ 1974 & 66.9 & 75.4 \\ 1975 & 67.4 & 75.9 \\ 1976 & 67.5 & 76.1 \\ 1977 & 67.9 & 76.7 \\ 1978 & 68.5 & 77.1\end{array}$

1 Including deaths due to war operations.

2 Excluding deaths due to war operations. 
Excess of births over deaths

$\begin{array}{lcr}\text { Number } & \begin{array}{r}\text { Per } 1000 \\ \text { of mean } \\ \text { population }\end{array} \\ 1901-10 & 38194 & 13.7 \\ 1911-20 & 24760 & 8.1 \\ 1921-30 & 28611 & 8.7 \\ 1931-40 & 20354 & 5.7 \\ 1941-50 & 44272 & 10.7 \\ 1951-60 & 48643 & 11.4 \\ 1961-70 & 32223 & 7.2 \\ 1971-75 & 16631 & 4.6 \\ 1975 & 21891 & 3.6 \\ 1976 & 22060 & 4.7 \\ 1977 & 21594 & 4.5 \\ 1978 & 20291 & 4.2\end{array}$

\section{International migration}

Emigrants who have obtained a passport

$$
\begin{aligned}
& 1901-10 \\
& 1911-20 \\
& 1921-30 \\
& 1931-40
\end{aligned}
$$$$
1941-50
$$$$
1951-60
$$$$
1961-70
$$$$
1971-75
$$$$
1976
$$$$
1977
$$$$
1978
$$$$
1979
$$

$$
\begin{array}{r}
150832 \\
67346 \\
58559 \\
8844
\end{array}
$$

Net migration

$$
\text { - } 41000
$$$$
\text { - } 70032
$$$$
-177626
$$$$
\begin{array}{r}
+\quad 9489 \\
\hline
\end{array}
$$

$\begin{array}{r}-\quad 9369 \\ \hline\end{array}$

- 10629

- 9403

- $6611^{*}$
Per cent of mean population
5.4
2.2
1.8
0.2

1.1

1.5

3.9

2.0

2.0

2.2

1.9

1.3 
Average in-migration to rural and urban municipalities

$1901-10$

$1911-20$

$1921-30$

1931-39

$1941-50$

$1951-60$

$1961-70$

$1971-75$

1976

1977

1978

1979

74784
85111
79248
136470
132778
172098
219330
233610
208946
188687
180804
$191573 *$

74784

79248

136470

132778

172098

219330

208946

188687

$191573 *$
Per cent of mean population

2.7
2.8
2.4
3.8
3.5
4.0
4.8
5.0
4.4
4.0
3.8
4.0

Economically active population by industry

$\begin{array}{lrrrr} & 1950 & 1960 & 1970 & 1975 \\ \begin{array}{lrrr}\text { Agriculture and forestry, hunting } \\ \quad\end{array} & 45.8 & 35.5 & 20.3 & 15.0 \\ \text { and fishing } & 20.8 & 21.6 & 25.9 & 27.2 \\ \text { Industry etc. } & 6.3 & 8.7 & 8.3 & 8.5 \\ \text { Construction } & 9.5 & 13.6 & 18.9 & 19.3 \\ \text { Tranmerce } & 5.4 & 6.3 & 7.1 & 7.6 \\ \text { Services } & 10.8 & 14.0 & 18.1 & 20.9 \\ \text { Unknown } & 1.4 & 0.3 & 1.4 & 1.5 \\ & 100.0 & 100.0 & 100.0 & 100.0\end{array}$

\section{Labor force participation rate of women}

Per cent of females in labor force

$\begin{array}{ll}1960-64 & 44.0 \\ 1965-69 & 43.3 \\ 1970-74 & 45.3 \\ 1975 & 46.5 \\ 1976 & 46.2 \\ 1977 & 46.6 \\ 1978 & 46.5 \\ 1979 & 46.9 *\end{array}$

